\title{
Les physiciens face au défi du réchauffement climatique
}

Le réchauffement climatique est une réalité. La courbe de variation de la température moyenne à la surface du globe depuis 1880 (fig. 1) est impitoyable. Elle est corroborée par la montée du niveau des mers due à la dilatation thermique des océans et à la fonte des glaciers continentaux.

Ce réchauffement représente une menace pour la vie sur Terre, à la fois directe par la progression de l'élévation des températures de l'atmosphère et des océans, par la montée des eaux et par les événements extrêmes qui en résultent (les canicules et les ouragans de ces dernieres années sont là pour nous le rappeler!), et indirecte par les sécheresses, famines, feux de forêts, difficultés d'adaptation des êtres vivants et leurs conséquences sur la biodiversité et les populations.

Aujourd'hui, tout montre que le réchauffement est d'origine anthropique : sa corrélation avec l'augmentation de la teneur en $\mathrm{CO}_{2}$ dans l'atmosphère, mémorisée dans les carottes glaciaires, son accroissement spectaculaire récent dû à l'émission de gaz à effet de serre par l'activité humaine, et sa coïncidence temporelle avec le développement industriel. Les modèles du climat en attestent de manière convergente. Réduire par un facteur important l'émission de gaz à effet de serre (consommer moins d'énergies fossiles, éviter la déforestation, tout en luttant contre la pauvreté, en dépit de l'augmentation prévisible de la population mondiale) va tester notre capacité collective à réagir (produire de l'énergie "décarbonée " et se montrer sobre et efficient) et notre capacité de résilience. C'est un défi majeur pour l'humanité.

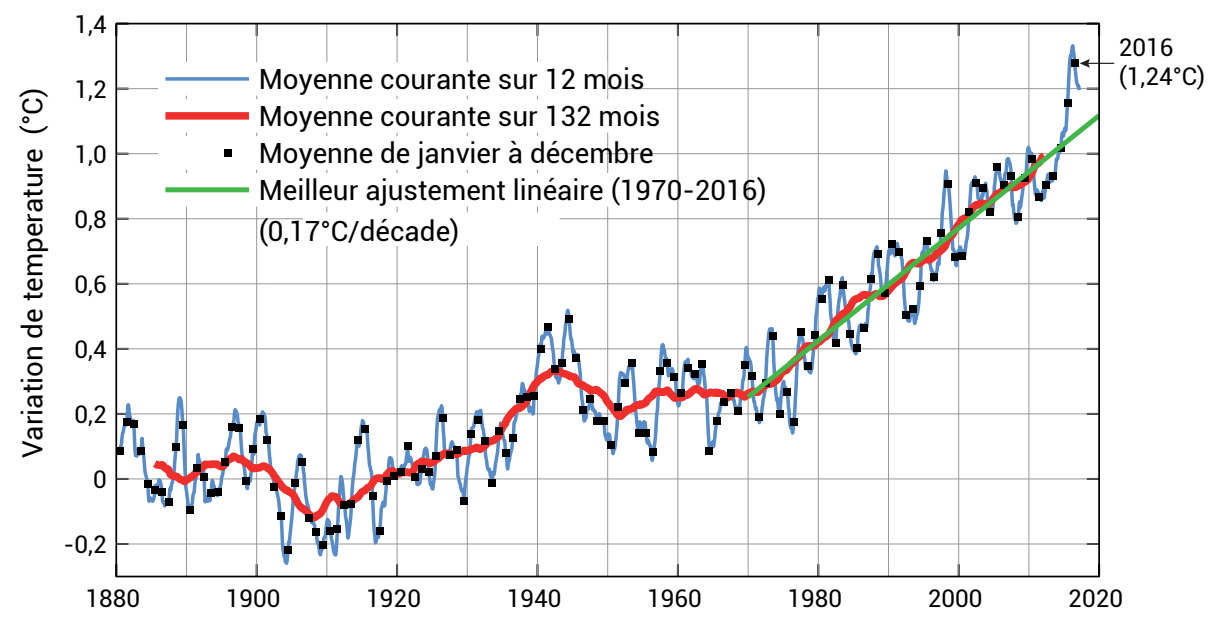

1. Variation de la température de surface globale de la Terre entre 1880 et 2016 , par rapport à la moyenne des années 1880 à 1920.

(c) Université de Columbia (www.columbia.edu/ mhs119/Temperature/), source NASA.

Voir aussi le blog de Sylvestre Huet du 17 août 2017 (huet.blog.lemonde.fr). 
Les sciences du climat sont devenues un domaine de recherches à part entière, multi et interdisciplinaire, dans lequel la physique joue tout son rôle au côté des autres disciplines. Le "modèle standard du climat " (voir http://wcrp-climate.org), partant des équations fondamentales de la dynamique pour déboucher sur des modèles numériques maillant le système Terre, avec ses différentes variantes, a bien progressé, même s'il reste beaucoup à faire (confronter, mieux cerner les acquis, les incertitudes et définir les points à approfondir). C'est une entreprise mondiale qui regroupe des milliers de chercheurs.

La physique contribue à fournir des pistes pour réduire l'émission de gaz à effet de serre dans l'activité humaine :

- en focalisant des recherches sur les sources d'énergie peu émettrices de $\mathrm{CO}_{2}$ (énergies dites décarbonées) : principe, mise en service, durabilité, coûts (y compris le coût en énergie et donc le taux de retour en énergie), impact du cycle (direct ou collatéral)...;

- en travaillant sur l'intermittence et le stockage (par exemple sur l'utilisation d'un parc généralisé de voitures hybrides, électriques et à bio-carburants, pour pallier à l'intermittence des énergies renouvelables, mais aussi sur de nouveaux moyens de stockage de l'énergie et sur des réseaux intelligents...) ;

- en travaillant, dans le cadre de la transition énergétique, sur l'optimisation dans la coexistence de l'énergie nucléaire et des énergies renouvelables;

- en travaillant sur la transformation éventuelle et/ou le stockage du $\mathrm{CO}_{2}$;

- en travaillant sur le concept de citoyen producteur et consommateur d'énergie ;

- en travaillant sur les économies d'énergie (processus), notamment en utilisant au mieux la chaleur (isolation, cycles combinés, réseaux de chaleur...) ;

- en conjuguant la transition énergétique et la lutte contre les inégalités entre populations humaines (notamment en donnant une chance à l'Afrique et à tous ceux qui ont le plus à perdre, et en prônant la sobriété énergétique...) ;

- en contribuant à alerter et aider le public, tous âges confondus, à comprendre les fondements scientifiques du climat et de ses enjeux, en veillant particulièrement à la jeunesse par une éducation adaptée, du primaire au supérieur. Les actions doivent s'accompagner d'une dimension d'espoir, convoquant dynamisme, goût d'agir et générosité (cf. projet d'un centre international d'aide aux enseignants, "International centre for climate change education", porté par l'ensemble des académies des sciences du monde et la fondation La main à la pâte [1]).

Un effort européen exceptionnel de recherche et développement, sur un mode coopératif, ouvert et stratégique, serait plus qu'opportun à ce stade. Il est temps de créer une Europe de l'énergie et du climat, comme il y a eu une Europe du charbon et de l'acier, ouverte sur le monde et notamment sur l'Afrique.

C'est le devoir des physiciens de s'associer aux climatologues (dont certains de nous font d'ailleurs partie). Pour affronter ce défi mondial à court, moyen et long terme, il faut lancer des alertes fondées scientifiquement, réfléchir en gardant l'esprit ouvert, développer et innover en partageant savoirs et savoir-faire à tous les niveaux (local, national, européen et mondial). En prenant exemple sur l'espérance de vie qui a partout augmenté grâce aux progrès de l'hygiène et de la médecine, il est temps de conjuguer nos efforts, citoyens, scientifiques et décideurs, pour faire reculer le réchauffement, les pollutions et les inégalités, bref, pour agir dans la direction d'un développement durable ! C'est notre meilleur moyen d'œuvrer pour la paix !

Michel Spiro

Président de la Société Française de Physique 